\title{
Braconidae (Hymenoptera) in the collection of the Institute of Zoology, NAS of Azerbaijan Republic Part IV. Subfamilies Orgilinae, Agathidinae, Ichneutinae, Cheloninae (Hymenoptera)
}

\author{
With 1 map \\ Arifa Abdinbekova ${ }^{1}$, Ellada Huseynova ${ }^{1,2}$ and Ilhama Kerimova 1,3

\begin{abstract}
${ }^{1}$ Institute of Zoology, NAS of Azerbaijan Republic, Az 1073, pr. 1128, bl. 504, Baku, Azerbaijan. - ${ }^{2}$ ella_line@mail.ru
3 ilkershah@hotmail.com

Published on 2015-06-30
\end{abstract}

\section{Summary}

The paper gives some faunistik records of the braconids from genera Orgilus, Charmon, belonging to subfamily Orgilinae, from genera Disophrys, Cremnops, Agathis, Bassus, Baeognatha, belonging to subfamily Agathidinae, from genus Pseudichneutes belonging to subfamily Ichneutinae and from genera Phanerotoma, Ascogaster, Chelonus belonging to subfamily Cheloninae. For each taxon localities in Azerbaijan, distribution in the world and hosts remarks are given. Some of the braconids below are located in the ZIN (Zoological Institute of the RAS), so the paper does not present information about them: 1. Orgilus (Orgilus) punctiventris ToBias, 1976; 2. Agathis syngenesiae NeEs, 1812; 3. A. glaucoptera Nees, 1834; 4. A. dzhulphensis Abdinbekova, 1970; 5. A. levis Abdinbekova, 1970; 6. A. caucasica ToвiAs, 1963; 7. A. transcaucasica Abdinbekova, 1970; 8. A. assimilis Kokuyev, 1895; 9. A. nachitshevanica Aвdinbekova, 1970; 10. Earinus nitidulus (Nees, 1812); 11. E. gloriatorius (Panzer, 1809); 12. Microdus punctatus (Aвdinbekova, 1970); 13. Proterops nigripennis Wesmael, 1835; 14. Ichneutes reunitor Nees, 1816; 15. Phanerotoma kozlovi Shestakov, 1930; 16. Phanerotoma atra Snoflak, 1951; 17. Phanerotoma gregori Snoflak, 1951; 18. Ascogaster kabystanica ToвiAs, 1976; 19. A. nachitshevanica AbDinBeKova, 1969; 20. A. klugii (NeEs, 1816); 21. Chelonus (Chelonus) tricolor Toвias, 1976; 22. Ch. (Ch.) subannulatus Abdinbekova, 1971; 23. Ch. (Ch.) subseticornis Toвias, 1971; 24. Ch. (M.) nachitshevanicus Aвdinвeкоva, 1971; 25. Ch. (M.) kiritshenko ToвiAs, 1976; 26. Ch. (M.) hungaricus Szepligeti 1896; 27. Ch. (M.) pectoralis ToBias, 1976; 28. Ch. (M.) talyshensis ToBias, 1976.

\section{Key words}

Hymenoptera, Braconidae, Orgilinae, Agathidinae, Ichneutinae, Cheloninae, collection, Azerbaijan

\section{Zusammenfassung}

In der Arbeit werden faunistische Daten der Bracconiden-Gattungen Orgilus und Charmon (Unterfamilie Orgilinae), Disophrys, Cremnops, Agathis, Bassus und Baeognatha (Unterfamilie Agathidinae), Pseudichneutes (Unterfamilie Ichneutinae) sowie Phanerotoma, Ascogaster und Chelonus (Unterfamilie Cheloninae) ausgewertet. Zu jedem Taxon werden Fundorte in Aserbaidschan, Verbreitung und Wirte genannt. Von folgenden Brackwespen-Arten aus der Sammlung des ZIN (Zoologisches Institut der RAS) fehlen jedoch diese Informationen: 1. Orgilus (Orgilus) punctiventris Tobias, 1976; 2. Agathis syngenesiae NeEs, 1812; 3. A. glaucoptera NeEs, 1834; 4. A. dzhulphensis AbDinbekova, 1970; 5. A. levis Abdinbekova, 1970; 6. A. caucasica Tobias, 1963; 7. A. transcaucasica Abdinbekova, 1970; 8. A. assi- 
milis Kokuyev, 1895; 9. A. nachitshevanica Abdinbekova, 1970; 10. Earinus nitidulus (NeEs, 1812); 11. E. gloriatorius (Panzer, 1809); 12. Microdus punctatus (Abdinbekova, 1970); 13. Proterops nigripennis Wesmael, 1835; 14. Ichneutes reunitor NeEs, 1816; 15. Phanerotoma kozlovi Shestakov, 1930; 16. Phanerotoma atra Snoflak, 1951; 17. Phanerotoma gregori SNoflak, 1951; 18. Ascogaster kabystanica ToвiAs, 1976; 19. A. nachitshevanica ABdinBeKovA, 1969; 20. A. klugii (Nees, 1816); 21. Chelonus (Chelonus) tricolor ToвiAs, 1976; 22. Ch. (Ch.) subannulatus Abdinbeкоva, 1971; 23. Ch. (Ch.) subseticornis Toвias, 1971; 24. Ch. (M.) nachitshevanicus Abdinbekova, 1971; 25. Ch. (M.) kiritshenko Tobias, 1976; 26. Ch. (M.) hungaricus Szepligeti 1896; 27. Ch. (M.) pectoralis Tobias, 1976; 28. Ch. (M.) talyshensis TOBIAs, 1976.

\section{Introduction}

Subfamily Orgilinae is presented by 5 species from 2 genera, subfamily Agathidinae by 19 species from 4 genera, subfamily Ichneutinae by 1 species from 1 genus and subfamily Cheloninae by 31 species from 3 genera in the entomological collection of the Institute of Zoology NAS of Azerbaijan. The paper includes distribution remarks and host range information for each species. The whole distribution areas in the world are provided for each taxon. Each name of species is accompanied by the author's name and description date. Map reference for the collection sites in Azerbaijan is presented. Pertinent literature was used for taxonomic analysis of the braconids (Marshall, 1900; Beyarslan et al., 2013; NieZabitowski, 1910; KoKujev, 1903a; KokUjev, 1903b; AвDINBEKova, 1965; 1967; 1971; 1975; 1995; WALTL, 1835; Tobias, 1971; 1986; Telenga, 1955; ERdoĞAN, 2013).

\section{Subfamily Orgilinae AsHMEAD, 1900}

\section{Genus Orgilus HaLIDAY, 1833}

\section{Subgenus Orgilus HALIDAY, 1833}

\section{Orgilus (Orgilus) nitidus MarShalL, 1898}

Material: dist. Nakhichevan AR, 25.V.1967, 1 \% , leg. Abdinbekova; dist. Goychay, 17.V.1964, 1 , leg. Abdinbekova.

Host: unknown.

Distribution: Azerbaijan, Hungary, Mongolia, Russia, Spain (MARSHALL, 1900).

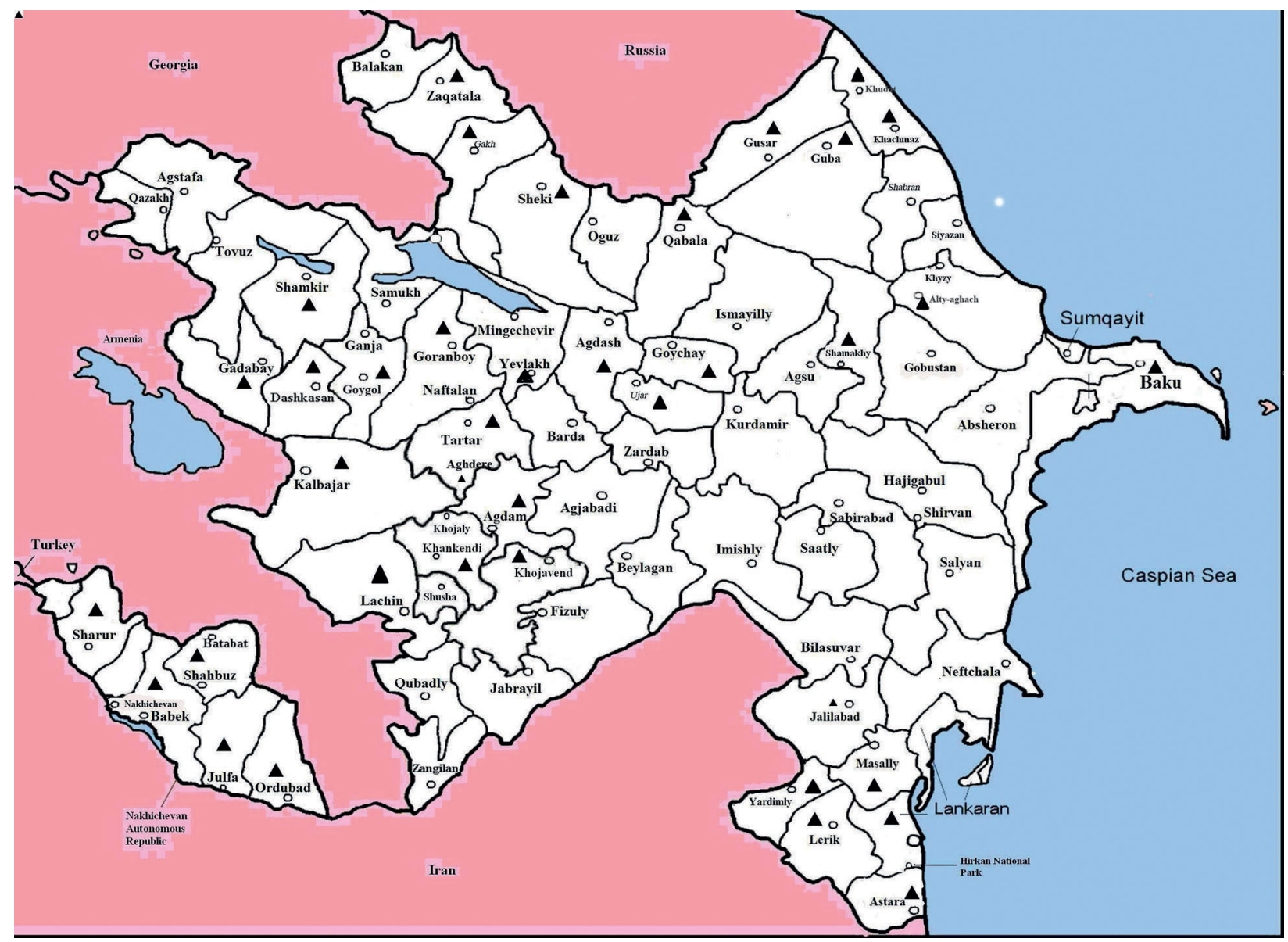

Map 1: Records of Bracconidae in Azerbaijan, places (open circles) and localities (filled triangles). 


\section{Orgilus (Orgilus) pimpinella NIEZABITOWsKI, 1910}

Material: dist. Guba, 6-7.VIII.1962, 3 ㅇ ㅇ, leg. Abdinbekova; dist. Lankaran, 27.IV.1965, 1 \% , leg. Abdinbekova; dist. Lerik, 30.VI.1965, 1 o , leg. Abdinbekova; dist. Margushevan (now Khojavend), 5.VIII.1966, 10 ㅇ ㅇ , 27 o o $o^{\star}$, leg. Abdinbekova; dist. Agdam, 5.VII.1966, 7 ㅇ ㅇ , leg. Abdinbekova; dist. Mardakert (now Aghdere), 8.VIII.1966, $7 \sigma^{\top} \sigma^{\top}$, leg. Abdinbekova; dist. Gasym-Ismayilov (now Goranboy), 7-11.VI.1966, $60^{\star} o^{\star}$, leg. Abdinbekova; dist. Kalbajar. 7.VIII.1966, 2 우, leg. Abdinbekova; dist. Shamkhor (now Shamkir), 19.VIII.1966, 2 우 우, leg. Abdinbekova; dist. Khankendi, 22.VIII.1966, $4 \sigma^{\top} \sigma^{\top}$, leg. Abdinbekova; dist. Gadabay, 28.VII.1966, 1 o , leg. Abdinbekova; dist. Khanlar (now Goygol), 8.IX.1966, 1 ㅇ, leg. Abdinbekova; dist. Ordubad, 5.VII.1967, 3 ㅇ 우 , leg. Abdinbekova; dist. Khachmaz, 15.VIII.1963, 1 ㅇ, leg. Abdinbekova; dist. Goychay, 7.VIII.1964, 1 ㅇ, leg. Abdinbekova; dist. Zagatala, 24.VII.1965, 1 ㅇ, leg. Abdinbekova; dist. Nukha (now Sheki), 26.V.1965, $10^{\star}$, leg. Abdinbekova; dist. Yardimly, 6.IX.1965, 1 ơ, leg. Abdinbekova.

Hosts: Agonopterix bipunctosa (CuRTIS, 1850) (Depressariidae), Anacampsis populella (ClerCK, 1759), A. temerella (Lienig \& Zeller, 1846), Argolamprotes micella (DENIS \& SCHIFFERMüLler, 1775), Athrips pruinosella (LIENIg \& Zeller, 1846), Caryocolum tricolorella (HAwORTH, 1812), Dichomeris juniperella (Linnaeus, 1761), Phthorimaea operculella (Zeller, 1873), Recurvaria nanella (DENIS \& SCHIFFERMÜLLER, 1775), Scrobipalpa (Euscrobipalpa) ocellatella (BoyD, 1858) (Gelechiidae), Coleophora discordella ZELLER, 1849, C. spiraeella ReBel, 1916, Depressaria Pimpinellae ZELLER, 1839 (Coleophoridae), Digitivalva arnicella (Heyden, 1863), (ACrolepiIdAe), Mompha (Lophoptilus) miscella (DENIS \& SCHIFFERMÜLLER, 1775), (Momphidae), Oncocera obductella Zeller, 1839 (Pyralidae), Rhyacionia buoliana DenIs \& SCHIFFERMÜLLER, 1775 (Tortricidae) (ToBIAs, 1986).

Distribution: Afghanistan, Austria, Bulgaria, Czech Republic, Germany, Greece, Hungary, Iran, Ireland, Italy, Kazakhstan, Korea, Lithuania, Moldova, Mongolia, Norway, Poland, Romania, Russia, Switzerland, Turkey, Ukraine, United Kingdom, Uzbekistan, Serbia, Palaearctic. Turkey (BeYArslan et al., 2013, Yu et al., 2012, Abdinbekova, 1975, Tobias, 1986).

\section{Orgilus (Orgilus) obscurator (NEES, 1812)}

Material: dist. Gusar, 11.VII.1960, 1 ๙, leg. Abdinbekova; dist. Nakhichevan AR, 25.V.1967, 1 \% , leg. Abdinbekova; dist. Lerik, 20.V.1965, 1 o , leg. Abdinbekova; dist. Shamakhy, 26.V.1966, 1 ㅇ, leg. Abdinbekova; dist. Guba, 1.VI.1962, 1 ơ, leg. Abdinbekova.
Hosts: Dendrolimus pini (Linnaeus, 1758) (Lasiocampidae), Agonopterix conterminella (ZELler, 1839), A. kaekeritziana (Linnaeus, 1767), (Depressariidae), Aproaerema anthyllidella (HüBNER, 1813), Dichomeris juniperella (LINNAEus, 1761), Exoteleia dodecella (Linnaeus, 1758), Loxostege sticticalis (Linnaeus, 1761), Recurvaria nanella (DENIS \& SCHIFFERMÜLLER, 1775), Scrobipalpa (Euscrobipalpa) acuminatella (SIRcom, 1850), S. (E) ocellatella (Boyd, 1858) (Gelechiidae), Coleophora alcyonipennella (KolLAR, 1832), C. discordella ZELLER, 1849 C. niveicostella ZelLer, 1839, C. paripennella Zeller, 1839, C. pyrrhulipennella Zeller, 1839 (Coleophoridae), Epinotia (Panoplia) cruciana (Linnaeus, 1761), Gypsonoma aceriana (DUPONCHEL, 1843), Lathronympha strigana (FABRICIUS, 1775), Rhyacionia buoliana (DENIS \& SCHIFFERMülleR, 1775), Rh. pinicolana (DoubledAY, 1849), Rh. pinivorana (Lienig \& ZELLER, 1846), Stictea mygindiana (DENIS \& SCHIFFERMüLleR, 1775), Tortrix viridana Linnaeus, 1758 (Tortricidae), Mompha epilobiella (DenIs \& SCHIFFERMüLleR, 1775), M. (Lophoptilus) miscella (Denis \& Schiffermüller, 1775), (Momphidae), Phalacropterix graslinella (BoISDUvAL, 1852) (Psychidae), Scythris picaepennis (HaworTH, 1828) (Scythridae), Yponomeuta evonymella (Linnaeus, 1758) (Yponomeutidae), Temelucha interruptor (GRAVENHORST, 1829) (Ichneumonidae), Hyssopus thymus Girault, 1916, (Eulophidae), Perilampus fulvicornis Ashmead, 1886, P. tristis Mayr, 1905 (Perilampidae), Andricus kollari (Hartig, 1843) (Cynipidae).

Distribution: Albania, Armenia, Austria, Azerbaijan, Belgium, Bulgaria, Canada, Chile, China, Croatia, Czech Republic, Finland, France, Germany, Hungary, Iran, Ireland, Italy (country), Kazakhstan, Latvia, Lithuania, Macedonia, Moldova, Mongolia, Netherlands, Norway, Poland, Russia, Slovakia, Slovenia, Sweden, Switzerland, Turkey, U.S.A, Ukraine, United Kingdom, Serbia (Yu et al., 2012, Авdinbekova, 1975, ToвiAs, 1986).

\section{Subgenus Ischiolus HeLléN, 1958}

\section{Orgilus (/schiolus) punctulator (NEES, 1812)}

Material: dist. Khachmaz, 7.VII.1961, $2 o^{\star} o^{x}$, leg. Abdinbekova; dist. Goychay, 14.VII.1964, 1 , leg. Abdinbekova; dist. Gadabay, 10.VII.1966, 1 \%, leg. Abdinbekova.

Hosts: Ancylis apicella Denis \& SCHIFFermüLler, (Tortricidae), Apterona crenulella (BRUAND, 1853), Megalophanes viciella (DENIS \& SCHIFFERMÜLLER, 1775) (Psychidae), Coleophora auricella (FABRICIUs, 1794), C. follicularis VAllot, 1802, C. galbulipennella ZELleR, 1838, C. nigricella STEPhens, 1835, C. saponariella HeEger, 1848, C. serratella Herrich-SChäfFer, 1855 (Coleophoridae), Yponomeuta malinellus Zeller, 1838, Y. padella (Linnaeus, 1758) (Yponomeutidae). 
Distribution: Armenia, Azerbaijan, Bulgaria, Croatia, Czech Republic, France, Germany, Hungary, Italy, Kazakhstan, Lithuania, Moldova, Mongolia, Netherlands, Poland, Russia, Sweden, Switzerland, Turkey, United Kingdom (Yu et al., 2012, AвDinbekova, 1975, Tobias, 1986).

\section{Genus Charmon HaLIDAY, 1833}

Subgenus Eubadizon NeEs, 1834

\section{Charmon (Eubadizon) extensor (LINNAEUS, 1758)}

Material: dist. Gusar, 23.VII.1960, 1 , leg. Abdinbekova.

Hosts: Saperda (Compsidia) populnea Linnaeus, 1758 (Chrysomeloidea), Dendroctonus micans (Kugelann, 1794) (Curculionoidea), Epirrita autumnata (BorkHAUSEN, 1794) (Geometridae), Operophtera bruceata (Hulst 1886), Acleris maccana (Treitschke, 1835), A. oxycoccana (PACKARD, 1869), A. rhombana (Denis \& SCHIFFERMÜLlER, 1775), A. variana (FERNALD, 1886), Ancylis comptana (Frolich, 1828), A. mitterbacheriana, A.rhombana (Denis \& SCHIfFERmüller, 1775, Apotomis semifasciana HaworTH, 1811, Archips rosana (Linnaeus, 1758), Argyrotaenia pinatubana (KearFOTt, 1905), A. tabulana Freeman 1944, Blastesthia posticana ZetTERStedt, 1839, Choristoneura diversana (HÜBNeR, 1817), Ch. fumiferana Clemens 1865, Ch. murinana HüBner, 1799, Ch. occidentalis Freeman, 1967, Ch. retiniana (WAlsingham, 1879), Cydia laricana (Busck, 1916), C. pomonella (Linnaeus, 1758), C. strobilella (LinnaEus, 1758), Epinotia lindana (FERNALD, 1892), Epinotia nigricana Herrich-SCHAFFER, 1851, Epinotia pusillana Peyerimhoff, 1863, Epinotia tedella (Clerck, 1759), Epinotia (Steganoptycha) tenerana Denis \& SCHIFFERMüller, 1775, Epinotia tetraquetrana HAworTH, 1811, Eupoecilia ambiguella (HüBNER, 1796), Exapate duratella Heyden, 1864, Grapholita molesta (Busck, 1916), Gravitarmata margarotana HeINEMANN, 1863, Hedya pruniana HüBner, 1799, Notocelia roborana Denis \& SChiffermüller, 1775, Pammene fasciana Linnaeus, 1761, Piniphila bifasciana Haworth, 1811, Ptycholomoides aeriferanus (Herrich-ScHAFfer, 1851), Rhyacionia buoliana (DeNIS \& SCHIfFERMÜLLER, 1775), Rhyacionia perangustana SNELlen, 1883, Spilonota lariciana HeInemanN, 1863, S. ocellana (Denis \& SCHIFFERMÜller,1775), Tortrix viridana (Linnaeus, 1758), Zeiraphera griseana HüBneR, 1799, Zeiraphera rufimitrana HerRICH-SCHAFFER, 1859 (Tortricidae), Agonopterix heracliana (Linnaeus, 1758), A. nervosa (HAwORTH, 1811), Depressaria pastinacella (Duponchel, 1838) (Depressariidae), Anacampsis populella (Clerck, 1759), Anarsia lineatella Zeller, 1839, Coleotechnites apicitripunctellus (Clemens 1860), C. atrupictellus (DiETZ, 1900) C. canusella (FrEemAN 1957), C. ducharmei (Freeman, 1962), C. milleri (Busck, 1914), C. piceaella (KeArfott, 1903), C. starki (Free-
MAN, 1957), C. thujaella (KeArfott, 1903), Gelechia hippophaella ZELler 1802, Teleiodes saltuum ZELLER, 1878, Monopis rusticella (HüBner, 1796) (Tineidae), Psoricoptera gibbosella (ZELLER, 1839) (Gelechiidae), Argyresthia goedartella (Linnaeus, 1758), A. (Blastotere) thuiella (PACKARD, 1871) (Yponomeutidae), Coleophora ulmifoliella McDunNough 1946 (Coleophoridae), Dioryctria abietivorella (GROTE, 1878) (Pyralidae), Hofmannophila pseudospretella (STAInton, 1849) (Oecophoridae), Stigmella poterii (STAInton, 1857) (Nepticulidae), Tischeria ekebladella (BJERKANDER, 1795) (Tischeriidae), Ypsolopha alpella DeNIs \& SCHIFFERMÜller 1775 (Ypsolophidae), Zygaena filipendulae (Linnaeus, 1758) (Zygaenidae), Earias clorana (Linnaeus, 1761), Mesapamea secalis (Linnaeus, 1758) (Noctuidae).

Distribution: Austria, Azerbaijan, Belgium, Bulgaria, Canada, China, Croatia, Cyprus, Czech Republic, Finland, France, Germany, Hungary, India, Ireland, Japan, Korea, Latvia, Lithuania, Mexico, Moldova, Mongolia, Netherlands, Norway, Poland, Portugal, Romania, Russia, Slovakia, Spain, Sweden, Switzerland, U.S.A., United Kingdom (Yu et al., 2012, АвDinbeKova, 1975, ToBias, 1986).

Subfamily Agathidinae HALIDAY, 1833

\section{Genus Disophrys FoERST, 1862}

\section{Disophrys dissors KoKUJEV, 1903}

Material: dist. Mardakert (now Aghdere), 21.VI.1967, 4 ㅇ, , leg. Abdinbekova.

Host: unknown.

Distribution: Azerbaijan, Hungary, Iran, Macedonia, Moldova, Turkmenistan, Ukraine, Serbia (KoKujev, 1903a; Kokujev, 1903b; Tobias, 1971, Авdinbekova, 1995).

\section{Disophrys inculcator LINNAEUS, 1758}

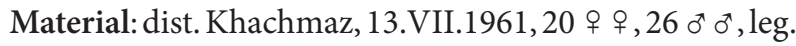

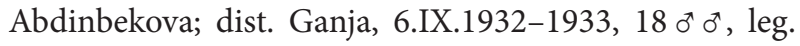
Unknown; dist. Gutkashen (now Gabala), 10.VIII.1935, 1 ơ, leg. Unknown; dist. Zagatala, 7.VIII.1960-1964, 14 우 ㅇ, leg. Abdinbekova; dist. Goychay, 6.X.1964,

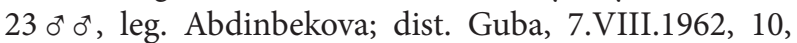
leg. Abdinbekova; dist. Masally, 8.VI.1965, 22 우 이, leg. Abdinbekova; dist. Gusar, 5.VIII.1961-1962, 9 우 우, leg. Abdinbekova; dist. Lerik, 20.VII.1965, $1 \sigma^{*}$, leg. Abdinbekova; dist. Lankaran, 5.IX.1965, $15 \sigma^{\star} \sigma^{\star}$, leg. Abdinbekova; dist. Yardimly, 7.IX.1965, 7 o $0^{\star}$, leg. Abdinbekova; dist. Margushevan (now Khojavend), 10.IX.1966, 
1 , , leg. Abdinbekova; dist. Agdam, 18.VII.1966, 1 \% 25.VIII.1966, 1 ㅇ, leg. Abdinbekova; dist. Ilich (now Babek), 21.IX.1967, 6 o $^{\star}$, dist. Ordubad, 13.IX.1967, 1 ㅇ leg. Abdinbekova; dist. Nakhichevan, 18.IX.1967, 1 o , leg. Abdinbekova; dist. Shamkhor (now Shamkir), 27.VII.1966, 1 o $^{\star}$, leg. Abdinbekova; dist. Kalbajar, 9.IX.1966, 1 o , leg. Abdinbekova; dist. Khankendi, 22.VIII.1966, 1 ơ, leg. Abdinbekova.

Host: unknown.

Distribution: Azerbaijan (Tobias, 1971; AbDinbekova, 1995), Hungary, Russia, Ukraine (Telenga, 1955).

\section{Disophrys caesia (KLUG, 1890)}

Material: dist. Masally, 4.VI.1965. 1 ㅇ (damaged), leg. Abdinbekova.

Host: unknown.

Distribution: Algeria, Armenia, Azerbaijan, Bulgaria Croatia, France, Germany, Hungary, Iran, Italy, Morocco, Portugal, Romania, Russia, Respublika, Serbia, Spain, Switzerland, Turkey, Serbia (Waltl, 1835; Tobias, 1971; Aвdinbekova, 1995; Telenga, 1955; Tobias, 1971).

\section{Genus Cremnops Forster, 1862}

\section{Cremnops desertor (LINNAEUS, 1758)}

Material: dist. Gusar, 14.VII.1960, 1 , leg. Abdinbekova

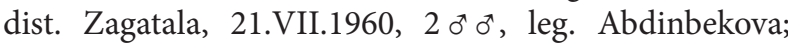
dist. Guba, 7.VIII.1962, 32 우 일 leg. Abdinbekova; dist. Shamkhor (now Shamkir), 24.VI.1966, 3 o o ๙ $^{\star}$ leg. Abdinbekova; dist. Lankaran, 3.V.1966, 1 ơ , 24.VI.1966, 1 ㅇ, leg. Abdinbekova; dist. Nukha (now Sheki), 26-27.V.1965, 2 ㅇ 우, leg. Abdinbekova; dist. Goychay, 5.VII.1964-1965, 2 우우, leg. Abdinbekova; dist. Khankendi, 28.VIII.1966, 1 ๙ , leg. Abdinbekova; dist. Margushevan (now Khojavend), 16.VIII.1966, $10^{*}$, dist. Goranboy, 16.VIII.1966, $1 \mathrm{o}^{\star}$, leg. Abdinbekova; dist. Gasym-Ismayilov (now Goranboy), 14.VII.1966, 2 우, 15.VIII.1966, 1 ㅇ , leg. Abdinbekova.

Hosts: Cydia pomonella (Linnaeus, 1758) (Tortricidae), Anania hortulata (Linnaeus, 1758) (Crambidae), Palpita machaeralis (WALKer, 1859) (Pyralidae), Synanthedon spheciformis (DENIS \& SCHIFFERMÜLLER, 1775) (Sesiidae), Perilampus microgastris Ferriere, 1930 (Perilampidae).

Distribution: Armenia, Azerbaijan, Belgium, Bulgaria, Canada, China, Croatia, Finland, France, Germany, Hungary, India, Indonesia, Italy, Japan, Korea, Latvia, Lithuania, Moldova, Myanmar, Nepal, Netherlands,
Poland, Romania, Russia, Serbia, Slovakia, Slovenia, Sweden, Switzerland, Turkey, U.S.A., Ukraine, United Kingdom (Tobias, 1986, Авdinbekova, 1995).

\section{Genus Agathis LATREILLE, 1804}

Agathis syngenesiae NeEs, 1814

Material: dist. Yardimly, 7.VI.1966, 1 ㅇ leg. Abdinbekova; dist. Gadabay, 10.VII.1966, 1 ㅇ, leg. Abdinbekova; dist. Masally, 27.VI.1965, 1 , leg. Abdinbekova.

Host: unknown.

Distribution: Azerbaijan, Croatia, Denmark, Finland, France, Germany, Greece, Hungary, Iran, Italy, Kazakhstan, Mongolia, Netherlands, Poland, Portugal, Russia, Spain, Sweden, Tajikistan, Turkey, Uzbekistan (Yu et al., 2012, АвDinbekova, 1975, TовiAs, 1986).

\section{Agathis unbellatarum NeES, 1812}

Material: dist. Mardakert (now Aghdere), 6-9.VI.1967, 3 우 우, leg. Abdinbekova; dist. Gusar, 14.VII.1962, 1 ơ, leg. Abdinbekova.

Hosts: Metzneria aestivella (Zeller, 1839), Metzneria lappella (Linnaeus, 1758) (Gelechiidae).

Distribution: Algeria, Azerbaijan, Bulgaria, Croatia, Cyprus, France, Germany, Greece, Hungary, Iran, Israel, Italy, Kazakhstan, Kyrgyzstan, Macedonia, Moldova, Mongolia, Portugal, Russia, Spain, Tajikistan, Tunisia, Turkey, Turkmenistan, Ukraine, Uzbekistan (ToвIAs, 1986, Авdinbekova, 1995).

\section{Agathis malvacearum LATREILLE, 1805}

Material: dist. Mardakert (now Aghdere), 6.VI.19661967, $30^{\top} o^{\star}, 2$ ㅇ 우, leg. Abdinbekova; dist. Guba, 29.VII.1960, 1 ㅇ, leg. Abdinbekova; dist. Gusar, VI.1960-1962, 2 우 우, dist. Margushevan (now Khojavend), 17.VI.1967, 1 \% , leg. Abdinbekova; dist. Kalbajar, 20.VII.1966, 2 ㅇ ㅇ , leg. Abdinbekova.

Hosts: Coleophora galbulipennella ZELLER, 1838, Coleophora graminicolella HeINEMANN, 1876 (Coleophoridae), Hellinsia didactylites STrom, 1783 (Pterophoridae), Metzneria aestivella Zeller, 1839, Metzneria lappella (Linnaeus, 1758), Pexicopia malvella HüBNER, 1805 (Gelechiidae), Rhyacionia resinella (Linnaeus, 1758) (Tortricidae), Perilampus fulvicornis Ashmead, 1886, Perilampus similis Crawford, 1914 (Perilampidae). 
Distribution: Albania, Armenia, Azerbaijan, Bulgaria, Canada, Croatia, Finland, France, Georgia, Germany, Greece, Hungary, Iran, Italy Kazakhstan, Latvia, Lithuania, Macedonia, Moldova, Mongolia, Netherlands, Poland, Romania, Russia, Slovakia, Slovenia, Spain, Baleares, Spain, Switzerland, Tajikistan, Turkey, U.S.A., Ukraine, United Kingdom, Uzbekistan, former Yugoslavia (ERDoĞAN, 2013).

\section{Agathis montana SHESTAKOV, 1932}

Material: dist. Alty-aghach, 11-12.VII.1965, 10 o o o $^{\star}$, leg. Abdinbekova.

Hosts: Pandemis cerasana (HüBner, 1786) (Tortricidae), Pyrausta aurata Scopoli, 1763 (Crambidae).

Distribution: Andorra, Armenia, Azerbaijan, Bulgaria, China, Czech Republic, France, Greece, Hungary, Iran, Kazakhstan, Korea, Kyrgyzstan, Macedonia, Moldova, Mongolia, Poland, Russia, Switzerland, Turkey, Ukraine, United Kingdom, Uzbekistan, Montenegro, Serbia (Yu et al., 2012, АвDinbeкova, 1975, Товias, 1986).

\section{Agathis semiaciculata Ivanov, 1899}

Material: dist. Masally, 7.IX.1965, 1 우 , leg. Abdinbekova.

Hosts: Coleophora onobrychiella ZELLER, 1849 (Coleophoridae).

Distribution: Azerbaijan, Bulgaria, China, Croatia, France, Georgia, Germany, Greece, Iran, Italy, Japan, Kazakhstan, Moldova, Mongolia, Poland, Russia, Switzerland, Turkmenistan, Ukraine (Yu et al., 2012, Aвdinbekova, 1975, Tobias, 1986).

\section{Agathis laticarpa TELENGA, 1955}

Material: dist. Gutkashen (Qabala), 14.VII.1960, 1 o , leg. Abdinbekova; dist. Guba, 13.V.1961, 1 \% , the same place 18.VII.1961, 2 o $o^{\star}$, leg. Abdinbekova; dist. Zagatala, 29.VII.1960, 1 o , leg. Abdinbekova.

Host: unknown.

Distribution: Armenia, Azerbaijan, Kazakhstan, Lithuania, Mongolia, Romania, Russia, Sweden, Ukraine (Yu et al., 2012, AвDinbekova, 1975, Tobias, 1986).

\section{Agathis duplicata SHESTAKOV, 1928}

Material: dist. Khachmaz, 10.X.1962, $1 \sigma^{*}$, leg. Abdinbekova; dist. Lerik, 30.VI.1965, 1 이 , leg. Abdinbekova; dist. Masally, 16.VII.1965, 1 ơ, leg. Abdinbekova.

Hosts: Coleophora granulatella Zeller, 1849 (Coleophoridae), Dichomeris marginella (FABRICIUS, 1781) Pexicopia malvella HüBNER, 1805, Scrobipalpa (Euscrobipalpa) ocellatella Boyd, 1858 (Gelechiidae).

Distribution: Armenia, Hungary, Mongolia, Poland, Yugoslavia, Serbia (Yu et al., 2012, AвDinbekova, 1975).

\section{Agathis genualis MARSHALL, 1898}

Material: dist. Masally, 5.IX.1965, 2 우 이 leg. Abdinbekova; dist. Gusar, 3.VII.1960, 1 o , leg. Abdinbekova; the same place, 22.VII.1962, $1 \sigma^{\star}$, leg. Abdinbekova; dist.

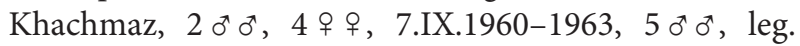
Abdinbekova; dist. Nukha (now Sheki), $30^{x} o^{x}, 4$ 우 우, 17.VII.1965, 2 우 일 leg. Abdinbekova; dist. Khanlar (now Goygol), 10.V.1966, 1 ㅇ, leg. Abdinbekova; dist. Lerik, 20.V.1965, 1 ㅇ , leg. Abdinbekova; dist. Ordubad, 5.VI.1965, 1967, 4 o $^{\star}$, leg. Abdinbekova; dist. Kirovabad (now Ganja), 1 우, 2 우 우, 20.VIII.1966, $3 \sigma^{x} o^{x}$, leg. Abdinbekova; dist. Lankaran, 5.IX.1965, 1 ○ै, leg. Abdinbekova; dist. Mirbashir (now Tartar), 15.VI.1967, 1 ㅇ , leg. Abdinbekova; dist. Kalbajar, 8.VI.1966, 1 o, leg. Abdinbekova, the same place, 23.VII.1966, 1 \%, leg. Abdinbekova, dist. Shamkhor (now Shamkir), 27.VII.1966, $1 \sigma^{\star}$, leg. Abdinbekova; dist. Nakhichevan, 6.IX.1967, $30^{\star} \sigma^{\star}$, leg. Abdinbekova; dist. Norashen (now Sharur), 23.V.1967, 1 ㅇ, leg. Abdinbekova; dist. Agdam, 22.VIII.1966, 1 o , leg. Abdinbekova; dist. Lachin, 28.VII.1966, 1 \% , leg. Abdinbekova; dist. Mardakert (now Aghdere), 20.VII.1966, 1 o*, leg. Abdinbekova; dist. Shahbuz, 14, 18.VI.1967, 10 우 우, leg. Abdinbekova; dist. Goranboy, 23.VI.1967, 1 ơ, leg. Abdinbekova; dist. Yardimly, 25.VI.1965, 1 ๙ , leg. Abdinbekova.

Host: unknown.

Distribution: Azerbaijan, Germany, Hungary, Kazakhstan, Kyrgyzstan, Lithuania, Moldova, Mongolia, Russia, Ukraine, Uzbekistan, Montenegro (Yu et al., 2012, Aвdinbekova, 1975, Toвias, 1986).

\section{Agathis breviseta NeES, 1812}

Material: dist. Guba, 17.VII.1962, 1 \% , leg. Abdinbekova.

Hosts: Aethes rutilana (HüBNER, 1817), Spilonota ocellana (DENIS \& SCHIFFERMüLler, 1775), Stictea mygindiana (Denis \& Schiffermüller, 1775) (Tortricidae), Agonopterix kaekeritziana LinnaEus, 1767, Chrysoesthia 
hermannella FABRICIUS, 1781, Monochroa cytisella (Curtis, 1837) (Gelechiidae), Coleophora HüBner, 1822, C. albitarsella Zeller, 1849, C. conyzae Zeller, 1868, C. follicularis VALLOT, 1802, C. inulae Wocke, 1876, C. lutipennella (ZELLER, 1838), C. trochilella DUPONCHEL, 1843 (Coleophoridae), Pyrausta purpuralis (Linnaeus, 1758) (Crambidae).

Distribution: Azerbaijan, Belgium, Bulgaria, Czech Republic, Czechoslovakia, Finland, France, Georgia, Germany, Greece, Hungary, Ireland, Italy, Kazakhstan, Latvia, Lithuania, Moldova, Mongolia, Netherlands, Poland, Russia, Slovakia, Slovenia, Sweden, Switzerland, Tajikistan, Turkey, Ukraine, United Kingdom (Yu et al., 2012, Авdinbekova, 1975, Toвias, 1986).

\section{Genus Bassus FabricIUS, 1804}

\section{Bassus rufipes (NEES, 1812)}

Material: dist. Khachmaz, 13.VII.1962, $1 o^{\star}$, leg. Abdinbekova; dist. Guba, 18.V.1963, 1 ơ, leg. Abdinbekova; dist. Khankendi, 22.VIII.1966, 1 우, leg. Abdinbekova.

Hosts: Acleris variegana (DENIS \& SCHIFFERMÜLLER, 1775), Apotomis capreana (HÜBNER, 1818), A. semifasciana (HAworth, 1811), Cacoecimorpha pronubana (HüBNer, 1799), Celypha lacunana Denis \& SCHIFFERMÜlleR, 1775, Cydia nigricana (FABRICIUs, 1794), Cydia pomonella (Linnaeus, 1758), Cydia funebrana (Treitschke, 1835), Grapholita molesta (BusCK, 1916), Gypsonoma dealbana (Frolich, 1828), Gypsonoma oppressana TREITsCHKe, 1835, Gypsonoma sociana Haworth, 1811, Hedya dimioalba Retzius, 1783, Hedya nubiferana (HAwORTH, 1811), Notocelia cynosbatella (Linnaeus, 1758), N. rosaecolana Doubleday, 1850, Pandemis cerasana (HüBNeR, 1786), Rhopobota ustomaculana CURTIS, 1831, Rhyacionia buoliana (DENIS \& SCHIfFERMÜLleR, 1775), Spilonota ocellana (Denis \& SCHIFFERMÜLleR, 1775), Tortrix viridana (LinnaEUS, 1758) (Tortricidae), Acrobasis consociella (HüBnER, 1813) (Pyralidae), Agonopterix ocellana FABricius, 1775 (Depressariidae), Anarsia lineatella Zeller, 1839, Caryocolum maculeum Haworth, Gelechia nigra HaworTH, 1828, Recurvaria leucatella (CLERCK, 1759), R. nanella (Denis \& Schiffermüller, 1775), Teleiodes luculella HüBNER, 1813 (Gelechiidae), Coleophora gryphipennella (HüBNER, 1796) (Coleophoridae), Yponomeuta evonymella (LinnAeus, 1758), Yponomeuta malinella Zeller, 1838, Yponomeuta padella (LinNAeus, 1758) (Yponomeutidae), Dibrachys microgastri (Bouche, 1834) (Pteromalidae), Perilampus tristis MAYr, 1905 (Perilampidae), Andricus kollari (Hartig, 1843), Biorhiza pallida Olivier, 1791 (Cynipidae).

Distribution: Armenia, Australia, Austria, Azerbaijan, Belgium, Bulgaria, Finland, France, Georgia, Germany,
Hungary, Italy, Japan, Kazakhstan, Korea, Kyrgyzstan, Lithuania, Moldova, Netherlands, Poland, Romania, Russia, Slovakia, Sweden, Switzerland, Turkmenistan, U.S.A., Ukraine, United Kingdom (Yu et al., 2012, ABDINBEKOVA, 1975, TоBIAs, 1986).

\section{Bassus mediator NeEs, 1812}

Material: dist. Guba, 25.VI.1962, 1 우, leg. Abdinbekova.

Hosts: Coleophora HüBner, 1822, C. anatipennella (HüBner, 1796), C. flavipennella (DuponCHeL, 1843), C. hemerobiella Scopoli, 1763, C. kuehnella Goeze, 1783, C. lutipennella (Zeller, 1838), C. pennella Denis \& SCHIFFERMÜLlER, 1775, C. serratella (LinNAEUS, 1761) (Coleophoridae), Scrobipalpa (Euscrobipalpa) ocellatella Boyd, 1858 (Gelechiidae).

Distribution: Armenia, Azerbaijan, Czech Republic, Finland, France, Germany, Hungary, Israel, Italy, Kazakhstan, Moldova, Netherlands, Poland, Russia, Sweden, Switzerland, Ukraine, United Kingdom (Yu et al., 2012, АвDinbekova, 1975, Товias, 1986).

\section{Bassus dimidiator (NeEs, 1834)}

Material: dist. Gusar, 22.VII.1960-1961, 3 ㅇ , $3 o^{\star \top} o^{\star}$, leg. Abdinbekova. dist. Guba, 15.VII.1961-1962-1963, $20^{\star \top} o^{\star}, 3$ 우 우, leg. Abdinbekova; dist. Goychay, 5 우 우, $7 o^{\star} o^{\star}, 3$.VIII.1964, 1 우 $4 o^{\star} o^{\star}$, leg. Abdinbekova.

Hosts: Anthonomus pomorum (Linnaeus, 1758) (Curculionidae), Acleris forsskaleana (LinnaEus, 1758), A. variana (FERNALD, 1886), Aleimma loeflingiana (Linnaeus, 1758), Archips cerasivorana (Fitch, 1856), Archips crataegana HüBNER, 1799, Archips rosana (Linnaeus, 1758), Archips xylosteana (Linnaeus, 1758), Argyrotaenia velutinana (WALKer, 1863), Choristoneura rosaceana (HARRIs, 1841) Croesia bergmanniana (Linnaeus, 1758), Cydia latiferreana (WAlsingham, 1879), Dichelia histrionana (Frolich, 1828), Epiblema scutulana Denis \& SCHIfFERMüLleR, 1775, Epinotia tetraquetrana HAWORTH, 1811, Grapholita interstinctana (Clemens, 1860), Grapholita molesta (Busck, 1916), Hedya nubiferana (HaworTH, 1811), Pandemis cerasana (HüBNER, 1786), Pandemis heparana DenIs \& SCHIFFERMÜLler, 1775 (Tortricidae), Blastodacna atra HAworth, 1828 (Agonoxenidae), Coleophora spinella SCHRANK, 1802 (Coleophoridae), Recurvaria leucatella (ClerCK, 1759), R. nanella (DENIs \& SCHIfFERMÜLLER, 1775) (Gelehiidae), Spilonota ocellana (DENIS \& SCHIfFERmüller, 1775), Tortrix viridana (Linnaeus, 1758), Yponomeuta malinella Zeller, 1838, Gelis tenellus (SAY, 1835), Scambus hispae (HARrIs, 1835) (Ichneumonidae). 
Distribution: Armenia, Azerbaijan, Belarus, Bulgaria, Canada, China, Finland, France, Germany, Greece, Hungary, Iran, Italy, Kazakhstan, Latvia, Lithuania, Moldova, Netherlands, Poland, Portugal, Russia, Slovakia, Switzerland, Turkey, U.S.A., Ukraine, United Kingdom, former Yugoslavia (Yu et al., 2012, ABDINBEкоVA, 1975, ТовiAs, 1986).

\section{Bassus tumidulus (NeES, 1812)}

Material: dist. Guba, 8, 9.V.1961-1962, 5 우 우, leg. Abdinbekova; dist. Nukha (now Sheki), 26.V.1965, 1 ơ $^{*}$ leg. Abdinbekova.

Hosts: Agonopterix atomella (Denis \& SCHIFFERMüLLER, 1775) (Depressariidae), Choristoneura fumiferana Clemens, 1865, Cydia compositella (FABricius, 1775), C. delineana Walker, 1863, C. pallifrontana Lienig \& Zeller, 1846, C. splendana HüBner, 1799, C. tenebrosana DüPONCHEL, 1843, Dichrorampha acuminatana LIENIG \& ZelLer, 1846, Epiblema cirsiana ZELLER, 1843, E. scutulana Denis \& SCHIFfermüller, 1775, Gypsonoma aceriana Duponchel, 1843, G. minutana HÜBNER, 1799, Lathronympha strigana FABRICIUs, 1775, Lobesia (Polychrosis) botrana (Denis \& SCHIFFERMÜLLER, 1775), L. (Lobesiodes) euphorbianus (FrEYER, 1840), Rhopobota ustomaculana CURTIS, 1831, Sparganothis pilleriana (DENIS \& SCHIFFERMÜLleR, 1775) (Tortricidae), Mompha epilobiella Denis \& Schiffermüller, 1775 (Momphidae), Ptocheuusa inopella (Zeller, 1839) (Gelechiidae), Utetheisa jacobaeae (LinnAeus, 1758) (Noctuidae).

Distribution: Austria, Azerbaijan, Belarus, Belgium, Bulgaria, China, Croatia, former Czechoslovakia, Finland, France, Georgia, Germany, Greece, Hungary, Iran, Ireland, Italy, Japan, Kazakhstan, Korea, Latvia, Lithuania, Moldova, Mongolia, Morocco, Netherlands, Norway, Poland, Portugal, Russia, Spain, Sweden, Switzerland, Turkey, Ukraine, United Kingdom, former Yugoslavia (Yu et al., 2012, AвDinbekova, 1975, Tobias, 1986).

\section{Genus Baeognatha KoKUJEv, 1903}

\section{Baeognatha niger (TELENGA, 1955)}

Material: dist. Goychay, 3.V.1964, 1 , leg. Abdinbekova.

Hosts: Coleophora flavipennella (DuponcheL, 1843), Coleophora lutipennella (Zeller, 1838) (Coleophoridae).

Distribution: Azerbaijan, former Czechoslovakia, France, Hungary, Kazakhstan, Poland, Russia, Slovakia, Switzerland, Ukraine (Yu et al., 2012, АвDInBEKovA, 1975, ToBias, 1986).

\section{Baeognatha armeniacus (TELENGA, 1955)}

Material: dist. Goranboy, 16.VIII.1966, 1 , leg. Abdinbekova; dist. Ordubad, 16.VII.1967, 1 \% , leg. Abdinbekova.

Hosts: Cydia funebrana (TReitschke, 1835) (Tortricidae), Anarsia eleagnella Kuznetzov, 1957, A. lineatella Zeller, 1839, Recurvaria leucatella (CLERCK, 1759), R. nanella (Denis \& Schiffermüller, 1775) (Gelechiidae).

Distribution: Armenia, Austria, Azerbaijan, Hungary, Iran, Italy, Kazakhstan, Moldova, Switzerland, Turkey, Ukraine (Yu et al., 2012, АвDinbekova, 1975, ToвiAs, 1986).

\section{Subfamily Ichneutinae FöRSTER, 1862}

Genus Pseudichneutes BELOKOBYLSKIJ, 1996

\section{Pseudichneutes levis WeSMAEL, 1835}

Material: dist. Guba, 12.V.1962, 1 ㅇ, leg. Abdinbekova.

Hosts: Fenusa dohrnii (Tischbein, 1846), Fenusa ulmi Sundevall, 1844, Metallus pumilus (Klug, 1814), Pontania proxima (LePeletier, 1823), Pontania viminalis (Linnaeus, 1758), Scolioneura vicina Konow, 1894 (Tenthredinidae).

Distribution: Belgium, Finland, France, Germany, Hungary, Italy, Kazakhstan, Netherlands, Poland, Russia, Sweden, Ukraine, United Kingdom (Yu et al., 2012, Авdinвeкоva, 1975, ТовiAs, 1986).

Subfamily Chelonina FöRSTER, 1862

Genus Phanerotoma WeSMAEL, 1838

\section{Phanerotoma acuminata SzÉPLIGETI, 1908}

Material: dist. Agdash, 2.VI.1960, 6 우, leg. Abdinbekova; dist. Goychay, 24.V.1964, 2 ㅇ ๑ , leg. Abdinbekova; dist. Lankaran, 14.VI.1966, 1 ๙, leg. Abdinbekova; dist. Lerik, 30.VI.1965, 1 , leg. Abdinbekova; dist. Masally, 15.VI.1965, 5 우 우, 8 o $^{\star}$, leg. Abdinbekova; dist. Guba, 3 우 우, 3 o $^{\star}$ o $^{\star}$, 8.IX.1961-1962, 3 leg. Abdinbekova.

Hosts: Hypsopygia costalis (FABRICIUs, 1775) (Pyralidae).

Distribution: Armenia, Austria, Azerbaijan, Canada, Czech Republic, France, Georgia, Germany, Hungary, Italy, Korea, Moldova, Mongolia, Netherlands, Romania, Russia, Switzerland, Turkey, U.S.A., Ukraine, United Kingdom, Serbia (Yu et al., 2012, Aвdinbekova, 1975, ToBIAs, 1986). 


\section{Phanerotoma minor ŠNOFLÁK, 1950}

Material: dist. Agdash, 13.VI.1961, 1 \%, leg. Abdinbekova.

Hosts: Lymantria dispar (LinNAeus, 1758) (Lymantriidae), Acrobasis consociella HüBnER, A. sodalella ZELleR, 1848 (Phycitidae).

Distribution: Azerbaijan, Russia, Kazakhstan, Czech Republic (Yu et al., 2012, AвDinbeKova, 1975).

\section{Phanerotoma planifrons (NEES, 1816)}

Material: dist. Nakhichevan, 19.VI.1967, 1 , leg. Abdinbekova; dist. Baku, 15.VII.1957, 1 ơ , leg. Abdinbekova.

Hosts: Dendrolimus punctatus (WALKer, 1855) (Lasiocampidae), Assara terebrella ZINCKen, 1818, Conobathra repandana FABRICIUs, 1798, Ephestia elutella (HüBNER, 1796), E. terebrellum ZELLER, Etiella zinckenella (Treitschke, 1832), Glyphodes pyloalis Walker, 1859, Phycita diaphana Staudinger, 1870 (Pyralidae), Cydia delineana WALKER, 1863, Cydia strobilella (LinnAEus, 1758), Cydia zebeana RATZEBURG, 1840, Grapholita molesta (BUSCK, 1916), Leguminivora glycinivorella (Matsumura, 1900) (Tortricidae), Diaphania indica (SAUnders, 1851), D. pyloalis WAlker, 1859, Haritalodes derogata (FABRICIUS, 1775) Crambidae.

Distribution: Afghanistan, Algeria, Azerbaijan, Belgium, Bulgaria, China, former Czechoslovakia, France, Georgia, Germany, Hungary, Italy, Japan, Kazakhstan, Korea, Moldova, Mongolia, Morocco, Portugal, Russia, Slovenia, Spain, Switzerland, Tunisia, Turkey, U.S.A., Ukraine, United Kingdom, Uzbekistan, Serbia (Yu et al., 2012, Abdinbekova, 1975, Tobias, 1986).

\section{Phanerotoma dentata (PANzer, 1805)}

Material: dist. Khachmaz, 21.VI.1962, 1 ㅇ, leg. Abdinbekova; dist. Norashen (now Sharur), 7.VIII.1962, $1 \sigma^{*}$, leg. Abdinbekova; dist. Goychay, 6, 7.VIII.1964, $50^{\star} \sigma^{\star}$, leg. Abdinbekova; dist. Masally, 5.VI.1965-1966, $70^{\star} o^{\star}$, leg. Abdinbekova; dist. Lankaran, 14.VI.1966, 1 ð, leg. Abdinbekova.

Hosts: Acrobasis consociella (HÜBNER 1813), Acrobasis sodalella ZELLER, 1848, Amyelois transitella (WALKER, 1863), Apomyelois ceratoniae (ZELLER, 1839), Conobathra tumidana Denis \& SCHIfFERMüLler, 1775, Etiella zinckenella (TREITschке, 1832), Trachycera advenella ZINCKEN, 1818 (Pyralidae), Carcina quercana (FABRICIUS, 1775) (Oecophoridae), Cydia funebrana (Treitschke, 1835), C. pomonella (Linnaeus, 1758), Epinotia (Panoplia) ramella (Linnaeus, 1758), Pammene amygdalana
Duponchel, 1842, Pammene gallicolana Lienig \& Zeller, 1846, Pammene populana (Fabricius, 1787), Pammene regiana Zeller, 1849, Phalonidia curvistrigana StAinton 1859, Rhopobota ustomaculana CuRTis, 1831, Spilonota ocellana (DENIS \& SCHIFFERMÜLLER, 1775), Zeiraphera isertana FABRICIUS, 1794 (Tortricidae), Gelechia turpella DenIs \& SCHIFFERMÜLlER, 1775 (Gelechiidae), Prays citri Milliere, 1873 (Yponomeutidae), Synanthedon andrenaeformis (LASPEYRES, 1801) (Sesiidae), Lymantria dispar (LinNAEus, 1758) (Noctuidae).

Distribution: Algeria, Argentina, Armenia, Austria, Azerbaijan, Belgium, Canary Islands, Croatia, Czechoslovakia, Egypt, Finland, France, Germany, Greece, Hungary, Italy, Kazakhstan, Kenya, Korea, Latvia, Lithuania, Moldova, Netherlands, Poland, Romania, Russia, Slovakia, Slovenia, Spain, Sweden, Switzerland, Ukraine, United Kingdom, Uzbekistan, former Yugoslavia (Yu et al., 2012, АвDinbekova, 1975, ToвiAs, 1986).

\section{Genus Ascogaster WeSMAEL, 1835}

\section{Ascogaster excisa (HeRRICH-Schaffer 1838)}

Material: dist. Kirovabad (now Ganja), 3.VIII.1966, 1 ㅇ, leg. Abdinbekova; dist. Guba, 18.VII.1962, 1 \% , leg. Abdinbekova; dist. Khanlar (now Goygol), 28.VIII. $10^{*}$, leg. Abdinbekova; dist. Shamakhy, 10.VI.1966, 7 o $^{\star} o^{\star}$, leg. Abdinbekova; dist. Shahbuz, 19, 22, 27.V.1967, 5 ㅇ 우, $4 \sigma^{\top} \sigma^{\star}$, leg. Abdinbekova; dist. Ordubad, 5.VI.1967, $3 \sigma^{\star} \sigma^{\star}$, leg. Abdinbekova; dist. Julfa, 5.VI.1967, 9 우 ㅇ, 2 o o o leg. Abdinbekova.

Host: unknown.

Distribution: Azerbaijan, Bulgaria, Croatia, France, Germany, Hungary, Italy, Kazakhstan, Moldova, Spain, Switzerland, Turkey (Yu et al., 2012, AвDINBEKovA, 1975, ToBIAs, 1986).

\section{Ascogaster annularis (NeEs, 1816)}

Material: dist. Guba, 14.VII.1962, 1 , leg. Abdinbekova.

Hosts: Batia lambdella (Donovan, 1793) (Oecophoridae), Chrysoclista linneella (CLERCK, 1759) (Agonoxenidae), Coleophora lutipennella (ZELler, 1838) (Coleophoridae), Narycia duplicella Goeze, 1783 (Psychidae), Pandemis cerasana (HüBner, 1786), Spilonota ocellana (Denis \& SChIfFermüller, 1775), Recurvaria leucatella (CLERCK 1759), R. nanella (DENIS \& Schiffermüller, 1775) (Tortricidae), Pandemis heparana Denis \& SChiffermüller, 1775, Parachronistis albiceps (ZELLER, 1839) (Gelechiidae), Stenolechia gemmella (Linnaeus, 1758) (Gelechiidae), Yponomeuta 
malinella ZeLLer, 1838 (Yponomeutidae), Y. padella (Linnaeus, 1758) (Yponomeutidae), Diplolepis rosae (Linnaeus, 1758) (Cynipidae).

Distribution: Armenia, Azerbaijan, Austria, Bulgaria, Czechoslovakia, Finland, France, Georgia, Germany, Hungary, Israel, Italy, Kazakhstan, Korea, Latvia, Lithuania, Moldova, Netherlands, Poland, Romania, Russia, Slovakia, Spain, Sweden, Switzerland, Turkey, Ukraine, United Kingdom (Yu et al., 2012, АвDinbeKova, 1975, ToBIAs, 1986).

\section{Ascogaster jaroslawensis KoKUYEV, 1895}

Material: dist. Guba, 5, 8.VII.1961-1962, 2 우 이, leg. Abdinbekova.

Hosts: Magdalis violacea (Linnaeus, 1758) (Curculionidae), Coleophora binderella (Kollar, 1832) (Coleophoridae), Cydia compositella (FABricius, 1775), C. grunertiana RAtZeBURG, 1868, C. pactolana ZelleR, 1840, C. zebeana Ratzeburg, 1840, Endothenia gentianaeana (HüBNER, 1799), Epinotia (Panoplia) cruciana (Linnaeus, 1761) (Tortricidae), Gelechia rhombella (Denis \& SCHIFFERMülLer, 1775) (Gelechiidae).

Distribution: Armenia, Azerbaijan, Kazakhstan, Lithuania, Moldova, Russia, Spain, Serbia (ToвiAs, 1986; AbdinbeKova, 1995).

\section{Ascogaster quadridentata WeSMAEL, 1835}

Material: dist. Zagatala, 17.VII.1959, 1 ㅇ, leg. Abdinbekova; dist. Khachmaz, 2 ㅇ , 3 o o đ̆ $^{*}$ 7.VII.1960-1963, 3 우 우, leg. Abdinbekova; dist. Guba, 5, 7, 9.VII.19611962, 11 ㅇ ㅇ, leg. Abdinbekova.

Hosts: Anthonomus pomorum (Linnaeus, 1758), Pachytychius strumarius Gyllenhal, 1836 (Curculionidae), Anthomyia albimana Zetterstedt, 1845 (Clusiidae), Clusiodes albimanus (Meigen, 1830) (Clusiidae), Andricus terminalis HARTIG, 1840, Biorhiza pallida Olivier, 1791 (Cynipidae), Eupithecia absinthiata (ClerCK, 1759), Operophtera brumata (Linnaeus, 1758) (Geometridae), Blastodacna atra HAwORTH, 1828, Bl. hellerella (Duponchel, 1838) Blastodacnidae), Adoxophyes orana Fischer von Roslerstamm, 1834, Aethes francillana (FABRICIUS, 1794), Ancylis achatana (Denis \& SChIfFermüller, 1775), Ancylis apicella Denis \& SChIfFermüller, 1775, Ancylis comptana (Frolich, 1828), Archips rosana (Linnaeus, 1758), Cnidocampa flavescens (WALKeR 1855), Croesia bergmanniana (Linnaeus, 1758), Cydia delineana WALKeR, 1863, C. duplicana Zetterstedt, 1839, C. funebrana (Treitschke, 1835), C. medicaginis Kuznetzov, 1962, C. nigricana (FABricius, 1794), C. pactolana Zeller,
1840, C. pallifrontana Lienig \& Zeller, 1846, C. pomonella (Linnaeus, 1758), C. splendana HÜBNER, 1799, C. strobilella (Linnaeus, 1758), C. zebeana RATzEBURG, 1840, Epinotia solandriana (LinnAEUs, 1758), Grapholita molesta (BuscK, 1916), G. packardi (ZELlER, 1875), G. prunivora (WALsh, 1868), Hedya nubiferana (HAWORTH, 1811), Lobesia (Polychrosis) botrana (Denis \& Schiffermüller, 1775), Lobesia (Polychrosis) viteana (Clemens, 1860), Notocelia cynosbatella (Linnaeus, 1758), Notocelia uddmanniana (Linnaeus, 1758), Olethreutes arbutella (Linnaeus, 1758), Pandemis cerasana (HüBNER, 1786), Pandemis heparana DENIS \& SCHIfFermüller, 1775, Spilonota ocellana (DENIS \& SCHIFFERMÜLler, 1775), Zeiraphera griseana HüBNER, 1799, Gypsonoma aceriana DuPONCHEL, 1843, G. dealbana (Frolich, 1828), G. oppressana Treitschke, 1835, G. sociana HAWORTH, 1811 (Tortricidae), Yponomeuta evonymella (Linnaeus, 1758), Yponomeuta malinella Zeller, 1838, Yponomeuta padella (Linnaeus, 1758) (Yponomeutidae), Desmia funeralis (HüBNER, 1796), Etiella zinckenella (TreItschKe, 1832), Loxostege sticticalis (LinnAeus, 1761), Diatraea saccharalis (FABRICIUS, 1794) (Pyralidae), Filatima persicaeella (MurTfelt, 1899), Recurvaria leucatella (CLERCK, 1759), Recurvaria nanella (DenIs \& SCHIFFERMüLlER, 1775) (Gelechiidae), Dibrachys cavus (WAlker, 1835), Pteromalus semotus (WALKer, 1834) (Pteromalidae), Dibrachys microgastri (Bouche, 1834), Perilampus Latreille, 1809, P. fulvicornis Ashmead, 1886, P. laevifrons Dalman, 1822, P. tristis MaYr, 1905 (Perilampidae), Phyllocolpa leucosticta (Hartig, 1837) (Tenthredinidae).

Distribution: Armenia, Austria, Azerbaijan, Belgium, Bulgaria, Canada China, Croatia, Cyprus, Czechoslovakia, Egypt, Finland, France, Germany, Greece, Hungary, Iran, Italy, Japan, Kazakhstan, Korea, Latvia, Lithuania, Macedonia, Madeira Islands, Moldova, Mongolia, Morocco, Netherlands, New Zealand, Peru, Poland, Romania, Russia, Slovakia, Spain, Sweden, Switzerland, Turkey, Turkmenistan, U.S.A., Ukraine, United Kingdom, Uzbekistan, Serbia (Yu et al., 2012, AвDinbekova, 1975, Toвias, 1986).

\section{Ascogaster rufipes (LATREILLE, 1809)}

Material: dist. Guba, 10.VIII.1961, 1 \%, leg. Abdinbekova; dist. Ordubad, 20.V.1967, 1 \% , leg. Abdinbekova; dist. Julfa, 21.V.1967, 1 ㅇ, leg. Abdinbekova.

Hosts: Anthonomus pomorum (Linnaeus, 1758) (Curculionidae), Blennocampa pusilla VittasaARi \& Vikberg, 1985 (Tenthredinidae), Coscinia cribraria (Linnaeus, 1758) (Arctiidae), Coleophora discordella Zeller, 1849, C. gryphipennella (HüBNER, 1796) (Coleophoridae), Agonopterix conterminella (ZELLER, 1839) (Depressariidae), Ethmia funerella (GöEzE, 1783) (Ethmiidae), Earias clorana (Linnaeus, 1761) (Nolidae), Acleris hasti- 
ana (Linnaeus, 1758), Archips oporana (Linnaeus, 1758), A. rosana (Linnaeus, 1758), Acleris holmiana (Linnaeus, 1758), Cydia funebrana (Treitschke, 1835), C. pomonella (Linnaeus, 1758), Dichelia histrionana (Frölich, 1828), Hedya nubiferana (HAwORTH, 1811), Lobesia botrana (DENIS \& SCHIFFERMÜLLER, 1775), Notocelia uddmanniana (LinnaEus, 1758), Olethreutes lediana (Linnaeus, 1758), Rhopobota ustomaculana (Curtis, 1831), Selania leplastriana (Curtis, 1831) (Tortricidae), Yponomeuta evonymella (Linnaeus, 1758), Y. padella (Linnaeus, 1758) (Yponomeutidae).

Distribution: Azerbaijan, Bulgaria, Kyrgyzstan, Russia, Turkey, Ukraine, Uzbekistan (Yu et al., 2012, ABDINBEKOVA, 1975).

\section{Genus Chelonus JURINE, 1801}

Subgenus Chelonus JURINE, 1801

Chelonus (Chelonus) bonellii (NeEs, 1816)

Material: dist. Gusar, 7.VIII.1962, 1 우, leg. Abdinbekova.

Host: unknown.

Distribution: Albania, Azerbaijan, Bulgaria, China, Croatia, Germany, Hungary, Italy, Kyrgyzstan, Macedonia, Russia, former Yugoslavia (Yu et al., 2012, Abdinbekova, 1975, Tobias, 1986).

\section{Chelonus (Chelonus) submuticus WeSMAEL, 1835}

Material: dist. Mingechevir, 22.IV.1947, 1 ㅇ, leg. Bogachev, dist. Guba, 1.VI.1962, 1 ơ, leg. Abdinbekova; dist. Masally, 27.V.1965, 1 ㅇ, leg. Abdinbekova; dist. Lankaran, 11.V.1966, $3 o^{\star} o^{\star}$, leg. Abdinbekova; dist. Gasym-Ismayilov (now Goranboy), 18.IV.1966, 1 ㅇ , leg. Abdinbekova; dist. Lerik, 15.V.1966, 1 ㅇ, leg. Abdinbekova; dist. Margushevan (now Khojavend), 21.V.1966, $2 o^{\star} o^{x}$, leg. Abdinbekova; dist. Mardakert (now Aghdere), 21.VI.1967, 1 우, leg. Abdinbekova.

Hosts: Homoeosoma nebulella (Denis \& SCHIFferMÜLLER, 1775) (Pylaiidae), Spodoptera littoralis (Boisduval, 1833) (Noctuidae), Adleria kollari (Hartig, 1843) (Cynipidae).

Distribution: Albania, Azerbaijan, Belgium, Bulgaria, Croatia, former Czechoslovakia, Finland, France, Georgia, Hungary, Israel, Italy, Kazakhstan, Macedonia, Moldova, Mongolia, Poland, Romania, Russia, Slovakia, Sweden, Switzerland, Ukraine, United Kingdom, Uzbekistan, Serbia (Yu et al., 2012, AвDINBEKovA, 1975, ToвIAs, 1986).

\section{Chelonus (Chelonus) oculator (FABRICIUs, 1775)}

Material: dist. Zagatala, 5, 7.VIII.1959-1960, 10 우, $12 \sigma^{\top} o^{\star}$, leg. Abdinbekova; dist. Astara, 25.VI.19651966, 8 우 우, 5 o $^{\star}$, leg. Abdinbekova; dist. Mingechevir, 22.IV.1940, 1 ơ, leg. Bogachev, dist. Guba, 4.VII.1962, 29 우 우, leg. Abdinbekova; dist. Gusar, 7.VII.1960-1962, 7 o $^{\star} \sigma^{\star}$, leg. Abdinbekova; dist. Khachmaz, 5.VIII.1962, 11 우 잉 leg. Abdinbekova; dist. Khudat, 16.VII.1961, $1 \mathrm{o}^{\star}$, leg. Abdinbekova; dist. Masally, 5, 7.IX.19631965, 50 우 우, 73 đ $^{\star}$ ๙ $^{\star}$, leg. Abdinbekova; dist. Goychay, 6.IX.1964, 19 우 우, leg. Abdinbekova; dist. Ujar, 13 o o $^{\star}$, 30.VI.1964, 2 o o , leg. Abdinbekova; dist. Yevlakh, 17.VI.1964, 4 ㅇ ㅇ , leg. Abdinbekova; dist. Devechi (now Shabran), 8.VII.1964, 1 ㅇ , leg. Abdinbekova; dist. Lerik, 6.VII.1965-1966, 8 o o $^{\star}$, leg. Abdinbekova; dist. Agdash, 13, 14.VII.1965, 5 ㅇ ㅇ , leg. Abdinbekova; the same place, 13, 23.VI.1966, 11 ơ $^{\top}$, leg. Abdinbekova; dist. Lankaran, 6.IX.1965-1966, 17 ㅇ ㅇ , leg. Abdinbekova; dist. Mardakert (now Agdere), 4-9.VI.1966-1967, 10 o $^{\top}{ }^{\top}, 6$ ㅇ , leg. Abdinbekova; dist. Kirovabad (now Ganja), 4.VI.19661967, $20^{\top} \sigma^{\star}$, leg. Abdinbekova; dist. Margushevan (now Khojavend), 5, 8.IX.1966, 7 , leg. Abdinbekova; dist. Shamkhor (now Shamkir), 24.VI.1966, $2 \sigma^{\top} \sigma^{*}$, leg. Abdinbekova; dist. Julfa, 13.VI.1967, 1 \% , leg. Abdinbekova; dist. Yardimly, 29.VI.1966, 1 ㅇ, leg. Abdinbekova; dist. Ordubad, 15.VI.1967, 1 우, leg. Abdinbekova.

Hosts: Agrotis segetum (DENIS \& SCHIFFERMÜLleR, 1775), Chortodes elymi (TreitschKe, 1825), Helicoverpa armigera (HüBNER, 1809), Heliothis peltigera (DENIS \& SCHIFFERMüLleR, 1775), Heliothis viriplaca (HüFNAGEL, 1766), Leucania loreyi (Duponchel 1827), Spodoptera exigua (HüBnER, 1808), Spodoptera littoralis (BoIsDuval, 1833) (Noctuidae), Coleophora anatipennella (HÜBNER, 1796) (Coleophoridae), Zeiraphera isertana FABRICIUS, 1794 (Tortricidae), Ephestia kuehniella Zeller 1879, Etiella zinckenella (TreitschKe 1832), Homoeosoma nebulella (Denis \& SCHiffermüLler, 1775) (Pyralidae), Loxostege sticticalis (Linnaeus, 1761), Ostrinia nubilalis (HüBNER, 1796) (Crambidae).

Distribution: Afghanistan, Albania, Azerbaijan, Belgium, Bulgaria, Canary Islands, China, Croatia, Czech Republic, Egypt, Finland, France, Georgia, Germany, Greece, Hungary, Iran, Italy, Kazakhstan, Latvia, Lithuania, Moldova, Mongolia, Netherlands, Norway, Poland, Romania, Russia, Slovakia, Slovenia, Spain, Sweden, Switzerland, Tajikistan, Turkey, Turkmenistan, Ukraine, United Kingdom, Uzbekistan, Serbia (Yu et al., 2012, Abdinbekova, 1975, Tobias, 1986).

\section{Chelonus (Chelonus) corvulus MarshaLl, 1885}

Material: dist. Dashkasan, 2.VIII.1966, 1 \%, leg. Abdinbekova; dist. Gadabay, 1 ㅇ, 3.VII.1967, 1 이, leg. 
Abdinbekova; dist. Guba, 17.VII.1967, 1 \% , leg. Abdinbekova.

Hosts: Chamaesphecia leucopsiformis (EsPER, 1800) (Sesiidae), Coleophora caespititiella ZELLER, 1839 (Coleophoridae), Dichrorampha simpliciana HaworTh, 1811 Epiblema foenella (Linnaeus, 1758) (Tortricidae), Scrobipalpa (Euscrobipalpa) atriplicella (FISCHER VON Roslerstamm, 1841) (Gelechiidae), Autographa gamma (Linnaeus, 1758), Eublemma pannonica (Freyer, 1840), Eublemma respersa (HüBNER, 1790), Shargacucullia verbasci (LinNAEUs, 1758), Spodoptera exigua (HüBNER, 1808) (Noctuidae).

Distribution: Azerbaijan, Belgium, China, Croatia, Czech Republic, Finland, France, Germany, Greece, Hungary, Israel, Italy, Kazakhstan, Latvia, Lithuania, Macedonia, Moldova, Mongolia, Netherlands, Poland, Romania, Russia, Slovakia, Slovenia, Spain, Switzerland, Turkey, Ukraine, United Kingdom, Uzbekistan, Serbia (Yu et al., 2012, АвdinbeKova, 1975, Tobias, 1986).

\section{Chelonus (Chelonus) caradrinae KokUJev, 1914}

Material: dist. Gusar, 14.VII.1961, 3 우 이 leg. Abdinbekova; dist. Guba, 7.IX.1961-1962, $290^{\pi} o^{*}$, leg. Abdinbekova; dist. Goychay, 6.VII.1964, $80^{\star} o^{\star}$, leg. Abdinbekova; dist. Ordubad, 6.IX.1967, 5 ㅇ 우, leg. Abdinbekova; dist. Agdash, 5 ㅇ ㅇ , 8.IX.1964-1966, 4 o ○ $^{*}$, leg. Abdinbekova; dist. Mardakert (now Agdere), 20-24.

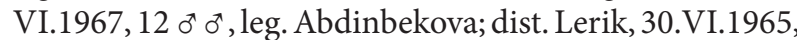
1 ㅇ, leg. Abdinbekova; dist. Khanlar (now Goygol), 23.V.1966, 1 \% , leg. Abdinbekova; dist. Margushevan (now Khojavend), 17.VII.1966, 1 ㅇ, leg. Abdinbekova; dist. Nakhichevan, 16-20.VI, IX.1967, 18 o $^{\star} o^{*}$, leg. Abdinbekova; dist. Astara, 25.VI.1965, 1 \% , leg. Abdinbekova; dist. Kalbajar, 3.IV.1966, 1 우, leg. Abdinbekova; dist. Shamkhor (now Shamkir), 24.VI.1966, $1 \sigma^{\star}$, leg. Abdinbekova; dist. Khachmaz, $10^{\star}$, 13.VII.1962, $20^{\star} o^{\star}$, leg. Abdinbekova.

Hosts: Spodoptera exigua (HüBNER 1808), Heliothis armigera (HüBNER, 1808) (Noctuidae).

Distribution: Azerbaijan, Croatia, Greece, Hungary, Italy, Kazakhstan, Moldova, Mongolia, Romania, Russia, Slovakia, Turkey, Turkmenistan, Ukraine, Uzbekistan, Serbia (Yu et al., 2012, АвDINBekova, 1975, TовiAs, 1986).

\section{Chelonus (Chelonus) shyrvanicus ABDInBEKOvA, 1967}

Material: dist. Devechi (now Shabran), $10^{\star}$, VII.1967, 5 우 오, leg. Abdinbekova; dist. Goychay, 28.V.1964, 1 우, leg. Abdinbekova; dist. Lerik, 30.VI.1965, 2 o $^{\text {đa }}$, leg. Abdinbekova.
Host: unknown.

Distribution: Azerbaijan, Serbia (AвDinbekovA, 1967).

\section{Chelonus (Chelonus) annulipes WeSMAEL, 1835}

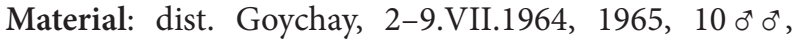
12 우 우, leg. Abdinbekova; dist. Guba, 20-30.VII.1960, 1961, 3 우, leg. Abdinbekova; dist. Khachmaz, 7.VIII.1962, 1963, 6 o $^{\top}$, leg. Abdinbekova; dist. Agdash, 14.VI.1964, 3 ○ oे $^{*}$ leg. Abdinbekova; dist. Masally, 6.IX.1965, 5 우, leg. Abdinbekova; dist. Astara, 6.VII.1965, 1966, 7 ○ o o $^{\star}$ leg. Abdinbekova; dist. Gasym-Ismayilov (now Goranboy), 5.VI.1966, 8 o $^{\star} 0^{\star}$, leg. Abdinbekova; dist. Margushevan (now Khojavend), 10.VI.1966, 1 ơ, leg. Abdinbekova, Astara, 6.VII.1965, 1966, 5 ㅇ ㅇ , leg. Abdinbekova; dist. Agdam, 8.VII.1966, 1 \% , leg. Abdinbekova; dist. Lachin, 22.VII.1966, 1 ơ , leg. Abdinbekova; dist. Nakhichevan, 5, 6.IX.1967, 16 o o o $^{*}$ leg. Abdinbekova; dist. Shahbuz, 27.V.1966, 1 o $^{\text {}}$, leg. Abdinbekova; dist. Julfa, 21.V.1966, 1 이 , leg. Abdinbekova; dist. Ordubad, 6.IX.1967, 2 ㅇ ㅇ , leg. Abdinbekova; dist. Ilich (now Babek), 21, 23.IX.1967, 10 , leg. Abdinbekova.

Hosts: Agrotis segetum (DeNIS \& SCHIFFERMÜLleR, 1775), Eublemma pannonica (Freyer, 1840), Heliothis viriplaca (Hufnagel, 1766), Spodoptera exigua (HüBNER, 1808), Porphyrinia pannonica Freyer, 1840 (Noctuidae), Ephestia kuehniella Zeller, 1879 (Pyralidae), Loxostege sticticalis (LINNAEUs, 1761), Ostrinia nubilalis (HüBNER, 1796) (Crambidae), Cydia pomonella (LinnAeus, 1758) (Tortricidae) Loxostege sticticalis (Linnaeus, 1761) (Crambidae).

Distribution: Azerbaijan, Kazakhstan, Iran, Mexico, Panama, Turkey, U.S.A. (Yu et al., 2012, AвdinbekovA, 1975, ToBIAs, 1986).

\section{Subgenus Microchelonus SzÉPLIGETI, 1908}

\section{Chelonus (Microchelonus) pusillus SzepLIGetı, 1908}

Material: dist. Guba, 30.VII.1961, 1 ㅇ, leg. Abdinbekova.

Host: Elachista Treitschke, 1833 (Elachistidae).

Distribution: Azerbaijan, Croatia, Czech Republic, Finland, Georgia, Hungary, Italy, Lithuania, Moldova, Mongolia, Poland, Russia, Spain, Ukraine, Serbia (Yu et al., 2012, Авdinbekova, 1975, Tobias, 1986). 


\section{Chelonus (Microchelonus) exilis MarshalL, 1885}

Material: dist. Gusar, 7, 14.VII.1960, 2 o $^{\star}$, leg. Abdinbekova; dist. Guba, 5.VIII.1960, 1962, 11 우, leg. Abdinbekova, dist. Gasym-Ismayilov (now Goranboy), 9.VI.1966, 1 ㅇ , leg. Abdinbekova; dist. Lerik, 30.VI.1965, 1 o', leg. Abdinbekova; dist. Goychay, 5.VI.1964, 1965, 4 우 우 , leg. Abdinbekova; dist. Ordubad, 5.VI.1967, $8 \sigma^{\star} \sigma^{\star}$, leg. Abdinbekova; dist. Khachmaz, 6.VIII.1966, $30^{\star} o^{\star}$, leg. Abdinbekova; dist. Julfa, 21.V.1967, 2 우 $0^{\star}$, leg. Abdinbekova; dist. Norashen (now Sharur), 23.V.1967, 3 우 우, leg. Abdinbekova; dist. Nakhichevan, 25, 28.V.1967, 5 우 우, leg. Abdinbekova; dist. Mirbashir (now Tartar), 5.VI.1967, $3 \circ^{\star} o^{\star}$, leg. Abdinbekova; dist. Mardakert (now Aghdere), 24.VIII.1966, 2 우 우, leg. Abdinbekova.

Hosts: Cosmopterix scribaiella ZELLER, 1850 (Cosmopterigidae), Elachista gangabella ZELLER, 1850 (Elachistidae), Lienigia lienigiella (ZELler, 1846) (Momphidae), Myelois circumvoluta Fourcroy, 1785 (Pyralidae).

Distribution: Armenia, Azerbaijan, Bulgaria, Croatia, France, Germany, Hungary, Italy, Kazakhstan, Latvia, Lithuania, Moldova, Mongolia, Netherlands, Poland, Russia, Slovenia, Spain, Switzerland, Turkey, United Kingdom, Uzbekistan, Serbia (Yu et al., 2012, AвDINBEKOVA, 1975, ТовіAs, 1986).

\section{Chelonus (Microchelonus) rostratus ToBIAs, 1966}

Material: dist. Shamakhy, 10.VI.1966, 2 ㅇ ㅇ , leg. Abdinbekova.

Host: unknown.

Distribution: Armenia, Azerbaijan, Bulgaria, Czech Republic, Hungary, Moldova, Russia, Slovakia, Spain, Turkey, Turkmenistan, Ukraine (Yu et al., 2012, ABDINBEKOVA, 1975, ToBias, 1986).

\section{Chelonus (Microchelonus) rimatus SzePLIGetı, 1896}

Material: dist. Guba, 7.VIII.1961, 1962, 5 우 우, Abdinbekova, dist. Goychay, 5 o $^{\top}$, 8.VIII.1964, 4 ㅇ 우, leg. Abdinbekova; dist. Masally, 5.VIII.1965, $190^{\star} \sigma^{\star}$, leg. Abdinbekova; dist. Zagatala, 20.V.1965, $1 \sigma^{\star}$, leg. Abdinbekova; dist. Astrakhan-bazar (now Jalilabad), 17.V.1965, 1 i , leg. Abdinbekova.

Hosts: Pexicopia malvella HüBner 1805 (Gelechiidae), Prays citri Milliere 1873 (Yponomeutidae).

Distribution: Armenia, Azerbaijan, Bulgaria, Croatia, Czech Republic, Hungary, Latvia, Lithuania, Moldova, Mongolia, Morocco, Poland, Spain, Switzerland, Ukraine,
Uzbekistan, Serbia (Yu et al., 2012, АвDinbekovA, 1975, ToBias, 1986).

\section{Chelonus (Microchelonus) telengai ABdinBeKova, 1965}

Material: dist. Devechi (now Shabran), 6.VII.1962, 6 우 오, leg. Abdinbekova; dist. Horadiz, 21.VI.1941, 1 우, leg. Yakovleva, dist. Mingechaur, 2.VII.1946, 1 \% , leg. Bogachev.

Host: unknown.

Distribution: Armenia, Azerbaijan, Iran (AвDInBekovA, 1965).

\section{Chelonus (Microchelonus) latrunculus Marshall 1885}

Material: dist. Goychay, 17.VII.1964, $10^{x}$, leg. Abdinbekova.

Host: Anthonomus pomorum (Linnaeus, 1758) (Curculionidae).

Distribution: Armenia, Azerbaijan, Czech Republic, Finland, Germany, Hungary, Italy, Moldova, Poland, Russia, Slovakia, Sweden, Switzerland, Tajikistan, Turkey, Ukraine, United Kingdom (Yu et al., 2012, AвDINBEKOVA, 1975, ToBias, 1986).

\section{Chelonus (Microchelonus) caucasicus AbdinBeKova 1967}

Material: dist. Yardimly, 6.IX.1965, 1 đ , leg. Abdinbekova; dist. Khachmaz, 15.V.1963, 1 , leg. Abdinbekova.

Host: unknown.

Distribution: Azerbaijan, Croatia, Greece, Hungary, Kazakhstan, Kyrgyzstan, Moldova, Russia, Spain, Switzerland, Turkey, Montenegro, Serbia (Yu et al., 2012, Abdinbekova, 1975, Tobias, 1986).

\section{Chelonus (Microchelonus) fenestratus NeEs, 1816}

Material: dist. Khachmaz, 20.V.1961, 1 ㅇ, leg. Abdinbekova; dist. Mardakert (now Aghdere), 26.VI.1967, 1 ㅇ leg. Abdinbekova; dist. Masally, 18.V.1965, 1 \%, leg. Abdinbekova; dist. Guba, 23.VI.1962, 1 ㅇ, leg. Abdinbekova.

Hosts: Dichrorampha alpinana (TREITSCHKe, 1830), D. petiverella (LinnaEus, 1758), Epiblema foenella (Linnaeus, 1758), Rhyacionia buoliana (Denis \& SchifFERMÜLLER, 1775) (Tortricidae). 
Distribution: Azerbaijan, Belgium, Czech Republic, Finland, France, Germany, Hungary, Ireland, Italy, Kazakhstan, Korea, Moldova, Mongolia, Poland, Romania, Russia, Slovakia, Slovenia, Turkey, Ukraine, United Kingdom, Uzbekistan, former Yugoslavia (Yu et al., 2012, Abdinbekova, 1975, Tobias, 1986).

\section{Chelonus (Microchelonus) nigritibialis ABDInBEKOvA, 1971}

Material: dist. Mardakert (now Aghdere), 21.IV.1966, 1 ㅇ, leg. Abdinbekova.

Host: unknown.

Distribution: Azerbaijan, Moldova, Turkey (AвDINBEKOVA, 1971).

\section{Chelonus (Microchelonus) contractus (NeEs, 1816)}

Material: dist. Khachmaz, 30.VII.1960, 1 ㅇ, leg. Abdinbekova; dist. Guba, 10.VII.1963, 1 ㅇ , leg. Abdinbekova; dist. Lerik, 20.VII.1965, 1 잉 leg. Abdinbekova; dist. Khankendi, 27.IV.1966, 1 o', leg. Abdinbekova; dist. Ordubad, 20, 26.V.1967, $3 \sigma^{\top} \sigma^{*}$, leg. Abdinbekova; dist. Shahbuz, 22, 30.V.1967, 2 ㅇ 우, 2 o $^{\top} 0^{\top}$ leg. Abdinbekova; dist. Norashen (now Sharur), 23.V.1967, 1 ơ $^{\star}$ leg. Abdinbekova.

Hosts: Anthophila fabriciana (Linnaeus, 1767) (Thyridoidea), Argyresthia pygmaeella (HüBNER, 1813) (Yponomeutoidea), Coleophora hungariae GozmánY, 1955 (Coleophoridae), Mirificarma mulinella Zeller, 1839, Phthorimaea operculella (ZELler, 1873) (Gelechiidae), Cydia nigricana (FABRICIUs, 1794), Rhyacionia buoliana (DENIS \& SCHIFFERMüLleR, 1775) (Tortricidae), Emmetia szoecsi KAsY, 1961 (Tischerioidea), Plutella xylostella (Linnaeus, 1758) (Yponomeutoidea), Prochoreutis myllerana (FABRICIUs, 1794) (Choreutidae), Stagmatophora extremella Wocke, 1871 (Cosmopteriginae).

Distribution: Armenia, Azerbaijan, Belgium, Bosnia Hercegovina, Bulgaria, Czech Republic, Finland, France, Germany, Greece, Hungary, Iran, Italy, Kazakhstan, Korea, Kyrgyzstan, Lithuania, Mongolia, Netherlands, Poland, Romania, Russia, Slovakia, Spain, Sweden, Switzerland, Turkey, Turkmenistan, Ukraine, United Kingdom, Serbia (Yu et al., 2012, АвdinbeKova, 1975, ToBias, 1986).

\section{Chelonus (Microchelonus) subcontractus ABDINBEKOVA, 1971}

Material: dist. Ordubad, 25.V.1967, 1 ㅇ, leg. Abdinbekova.

Hosts: Elachista Treitschke, 1833 (Elachistidae), Phthorimaea operculella (ZELleR, 1873) (Gelechiidae), Stagmatophora extremella Wocke, 1871 (Cosmopteriginae).

Distribution: Azerbaijan, Georgia, Greece, Hungary, Kazakhstan, Lithuania, Moldova, Mongolia, Poland, Romania, Russia, Slovakia, Turkey, Ukraine, Serbia (YU et al., 2012, Aвdinbekova, 1975, Toвias, 1986).

\section{Chelonus (Microchelonus) azerbajdzanicus ABDINBEKOVA, 1971}

Material: dist. Astara, 17, 25.VI.1965, $2 \sigma^{\top} \sigma^{-}$, leg. Abdinbekova.

Host: unknown.

Distribution: Armenia, Azerbaijan, Greece, Spain.

\section{Chelonus (Microchelonus) rugicollis Th0ms, 1874}

Material: dist. Guba, 8, 9.V.1962, 2, leg. Abdinbekova; dist. Khankendi, 27.IV.1966, 1, leg. Abdinbekova (Yu et al., 2012, Abdinbekova, 1975, Tobias, 1986).

Host: Alucita hexadactyla (Linnaeus, 1758) (Alucitidae).

Distribution: Albania, Azerbaijan, Hungary, Slovakia, Spain, Sweden, former Yugoslavia (Yu et al., 2012, AbdinbeKova, 1975, Tobias, 1986).

\section{Chelonus (Microchelonus) magnifissuralis ABDINBEKOVA, 1971}

Material: dist. Shahbuz, 27.V.1967, $10^{\star}$, leg. Abdinbekova; dist. Margushevan (nov Kojavend), 28.IV.1966, 1 đ, leg. Abdinbekova; dist. Julfa, 25.V.1967, 1 o', leg. Abdinbekova.

Host: unknown.

Distribution: Azerbaijan, Spain, Turkey (Yu et al., 2012, Aвdinbekova, 1975, Товias, 1986). 
Chelonus (Microchelonus) alboannulatus SzePLIGETI, 1896

Material: dist. Khachmaz, 5, 7.VIII.1960, 1961, 3 o $^{\star} 0^{\star}$, leg. Abdinbekova; dist. Mardakert (now Aghdere), 6.VIII.1966, $40^{\top} 0^{\top}$, leg. Abdinbekova; dist. Gusar, 7.VIII.1961, $30^{\widehat{ }} 0^{\text {, }}$, leg. Abdinbekova; dist. Guba, 7.VIII.1962, 4 우 우, 5 đ̊ $^{\top}$, leg. Abdinbekova; dist. Goychay, 12.VII.1964, $30^{\text {ते }} 0^{\text {, }}$, leg. Abdinbekova; dist.

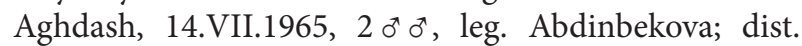
Yardimly, 29.VI.1966, 1 o , leg. Abdinbekova.

Hosts: Loxostege sticticalis (Linnaeus, 1761), Ostrinia nubilalis (HüBNER, 1796), (Crambidae), Exeristes roborator (FABricius, 1793) (Ichneumonidae), Pteromalus poecilopus (CraWford, 1910) (Pteromalidae), Chamaesphecia euceraeformis (OCHSENHEIMER, 1816) (Sesiidae).

Distribution: Armenia, Azerbaijan, Bulgaria, former Czechoslovakia, Hungary, Kazakhstan, Lithuania, Moldova, Russia, Switzerland (Yu et al., 2012, AвDInBEKOVA, 1975, TовIAS, 1986).

\section{References}

Aвdinbekova, A. A. 1965: New species and forms of insects (Fam. Ichneumonidae and Braconidae) from Kuba-Khachmask zone in Azerbaidjan. - Doklady Akademii Nauk Azerbaidzhanskoi SSR. 21 (4): 61-64 (in Russian).

Abdinbekova, A. A. 1967: New species of braconids (Braconidae, Hymenoptera) from the fauna of Azerbaidzhan. - Doklady Akademii Nauk Azerbaidzhanskoi SSR. 23 (10): 50-53 (in Russian).

Abdinbekova, A. A. 1971: Braconids of the genus Chelonus Jurine (Hymenoptera, Braconidae) of Azerbaidzhan. - Entomologicheskoye Obozreniye. 50 (2): 392-403. - Entomological Review 50: 221-228.

Abdinbekova, A. A. 1975: Braconids (Hymenoptera, Braconidae) of Azerbaijan. - Baku: 324 pp. (in Russian).

Aвdinbekova A. A. 1995: Hymenopterans (Hymenoptera, Braconidae) of Azerbaijan. - Baku, 472 pp. (in Azery).

Beyarslan, A.; GözüaÇIK, C. \& Inanc, Ö. 2013: A contribution on the subfamilies Helconinae, Homolobinae, Macrocentrinae, Meteorinae, and Orgilinae (Hymenoptera: Braconidae) of southeastern Anatolia with new records from other parts of Turkey. Turkish Journal of Zoology 37: 501-505.

ERdoĞan Ö. Ç. 2013: Contributions to the knowledge of Agathidinae fauna of the Eastern Anatolia Region of Turkey. - Turkish Journal of Zoology 37: 195-199.
KokUjev, N. R. 1903a: Ichneumoniden und Braconiden aus den Iran 1954 (Hymenoptera). - Jahresheft des Vereins für Vaterlaendische Naturkunde $112(1)$ : 103-117.

Kokujev, N. R. 1903b: New Transcaspian species of the subfamily Agathidinae (Hymenoptera, Braconidae). - Trudy Russkago Entomologicheskago Obshchestva. Horae Societatis Entomologicae Rossicae 36: 240-247.

Marshall, T. A. 1900: Les Braconides (Supplement). - In: ANdre, E. (ed.) 1897-1900. 'Species des Hymenopteres d'Europe et d'Algerie.' Paris. Tome 5: $369 \mathrm{pp}$.

NiezABITOwski, E. L. 1910: Materyaly do fauny Brakonidow Polski. Braconidae, zebrane w Galicyi. Sprawozdania Akademii Umiejetnosci w Krakowie. 44: 47-106.

Telenga, N. A. 1955: Braconidae, subfamily Microgasterinae, subfamily Agathinae. Fauna USSR, Hymenoptera 5 (4): 311 pp. - Translated from Russian by Israel Program for Scientific Translation, Jerusalem, 1964.

Tobias V. I. 1971: Obzor nayezdnikov - brakonid fauny SSSR. - Press AUES 54: 156-268.

Tobias V. I. 1986: Key for insects of European part of SSSR. v. III. Hymenoptera. IV. Press. Nauka: 500 pp.

WaltL, J. 1835: Reise durch Tyrol, Oberitalien und Piedmont nach dem sudlichen Spanien. Zweiter Theil. - Ueber die Thiere Andalusiens, Passau: 120 pp.

Yu, D. S.; Van Achterberg, K. \& Horstmann, K. 2012: World Ichneumonoidea 2005. Taxonomy, biology, morphology and distribution [Braconidae]. - Taxapad 2006 (Scientific names for information management) Interactive electronical catalogue on DVD/CD-ROM. Vancouver. 